\title{
Special issue on selected papers from NORCHIP 2013 conference
}

\author{
Romualdas Navickas
}

Published online: 8 August 2014

(c) Springer Science+Business Media New York 2014

This special issue of Analog Integrated Circuits and Signal Processing presents the extended versions of selected papers from the 31th NORCHIP conference which was held in Vilnius, Lithuania, November 11-12, 2013.

The 31th NORCHIP conference had 56 registered participants and 45 papers were presented during the 2 days the conference lasted whereof 30 papers were presented in 10 oral lecture sessions in two tracs, 12 papers were presented in two poster sessions, the 3 presentation, in session on Education and Training. The 3 invited presentations (first-Romualdas Navickas, "Self-formation in high speed integrated circuits", second-Petar Popovski, "Wireless technologies competing for a seat in the 5G bandwagon", and third-Ville Eerola, "Correlator design and implementation for GNSS receivers") were given. However, more submissions are originating from the local area of the conference (only one accepted paper was from a non-European country).

From the contributed papers nine papers have been selected for this special issue. The selection of the papers for this special issue is based on quality criteria and they reflect the topics in analog circuit design highlighted during the conference, the feedback of the session chairs and the selected papers all contain contributions that will be of interest to the reader. The papers are briefly introduced below.

The two first papers present two RF amplifiers. In the first paper "Triple Cascaded Current-Reuse Low Noise Amplifier" Muh-Dey Wei, Sheng-Fuh Chang, Renato Negra present a triple cascaded current-reuse CMOS low noise amplifier (LNA) for $3.5 \mathrm{GHz}$ WiMAX application. Three common-

\section{R. Navickas $(\square)$}

Department of Computer Engineering, Vilnius Gediminas

Technical University, Naugarduko str. 41, r. 437, 03227 Vinius, Lithuania

e-mail: romualdas.navickas@vgtu.lt source amplifiers are stacked and reuse the same current. This triple cascaded topology is able to enhance power gain but needs two coupling networks which costs enormous chip size. In order to have reasonable chip size, two coupling methods are investigated. For obtaining simultaneous input and noise matching, an additional capacitor is employed to adjust quality factor and reduce the gate induced current noise. The proposed chip is implemented in $180 \mathrm{~nm}$ CMOS technology. The measurement results show a maximum power gain of $21.7 \mathrm{~dB}$ and minimum noise figure of $3.11 \mathrm{~dB}$. The chip size is $1.05 \times 0.93 \mathrm{~mm}$ including all pads and the power consumption is $5.16 \mathrm{~mW}$ with a supply voltage of $1.5 \mathrm{~V}$.

In the second paper "A $1 \mathrm{~V}$ Power Amplifier for 81-86 GHz E-band” authors Tobias Tired, Henrik Sjöland, Carl Bryant and Markus Törmänen describe the design and layout of a two stage SiGe E-band power amplifier using a stacked transformer for output power combination. In EMsimulations with ADS Momentum, at E-band frequencies, the power combiner consisting of two individual single turn transformers performs significantly better than a single 2:1 transformer with two turns on the secondary side. Imbalances in the stacked transformer structure are reduced with tuning capacitors for maximum gain and output power. At $84 \mathrm{GHz}$ the simulated loss of the stacked transformer is as low as $1.35 \mathrm{~dB}$, superseding the performance of an also presented alternative power combiner. The power combination allows for a low supply voltage of $1 \mathrm{~V}$, which is beneficial since the supply can then be shared between the power amplifier and the transceiver, thereby eliminating the need of a separate voltage regulator. To improve the gain of the two-stage amplifier it employs a capacitive cross-coupling technique not yet seen in mm-wave SiGe Pas.

Capacitive cross-coupling is an effective technique for gain enhancement but is also sensitive to process variations as shown by Monte Carlo simulations. To mitigate this two 
alternative designs are presented with the cross coupling capacitors implemented either with diode coupled transistors or with varactors. The PA is designed in a SiGe process with $\mathrm{f}_{\mathrm{T}}=200 \mathrm{GHz}$ and achieves a power gain of $12 \mathrm{~dB}$, a saturated output power of $16 \mathrm{dBm}$ and a $14 \%$ peak PAE. Excluding decoupling capacitors it occupies a die area of $0.034 \mathrm{~mm}^{2}$.

The second group of papers from 3 articles concern data conversion integrated circuits. In the third paper "A 7-bit $40 \mathrm{MS} / \mathrm{s}$ Single-ended Asynchronous SAR ADC in $65 \mathrm{~nm}$ CMOS" authors $\mathrm{Ye} \mathrm{Xu}$ and Trond Ytterdal presents a 7-bit $40 \mathrm{MS} / \mathrm{s}$ single-ended asynchronous SAR ADC intended for in-probe ultrasound imaging systems. A single-ended architecture is proposed for a moderate resolution for its simplicity. Together with a double reference technique, the architecture reduces the area of the technology-limited large capacitors. The speed is optimized by an asymmetric delay line embedded in the asynchronous digital logic, enabling a sampling frequency of $40 \mathrm{MS} / \mathrm{s}$. The prototype is fabricated in a $65 \mathrm{~nm}$ CMOS technology. Measurement shows that at $1 \mathrm{~V}$ supply and $40 \mathrm{MS} / \mathrm{s}$, the ADC achieves an SNDR of $39.73 \mathrm{~dB}$ and an ENOB of 6.3 bit, while consuming $298.6 \mu \mathrm{W}$, resulting in an energy efficiency of $94.74 \mathrm{fJ} /$ conversion-step.

In the fourth paper "Design and Analysis of High Speed Capacitive Pipeline DACs" authors Tai Quoc Duong, Jerzy J. Dąbrowski and Atila Alvandpour are proposing design of a high speed capacitive digital-to-analog converter (SC DAC) for $65 \mathrm{~nm} \mathrm{CMOS} \mathrm{technology.} \mathrm{SC} \mathrm{pipeline} \mathrm{architecture} \mathrm{is} \mathrm{used}$ followed by an output driver. For $\mathrm{GHz}$ frequency operation with output voltage swing suitable for wireless applications (300 $\mathrm{mVpp}$ ) the DAC performance is shown to be limited by the capacitor array imperfections. While it is possible to design a highly linear output driver with HD3 $<-70 \mathrm{~dB}$ and HD2 $<-90 \mathrm{~dB}$ over $0.5-5 . \mathrm{GHz}$ band as we show, the maximum SFDR of the SCDAC is $45 \mathrm{~dB}$ with 8-bit resolution and Nyquist sampling of $3 \mathrm{GHz}$. The capacitor array is designed based on the DAC design area defined in terms of the switch size and unit capacitance value. A tradeoff between the DAC bandwidth and resolution accompanied by SFDR is demonstrated. High linearity of the output driver is attained by a combination of two techniques, the derivative superposition (DS) and resistive source degeneration. In simulations the complete DAC achieves SFDR of $45 \mathrm{~dB}$ with 8-bit resolution for signal bandwidth $1.36 \mathrm{GHz}$ with Nyquist sampling. With 6-bit and $5.5 \mathrm{GHz}$ bandwidth $33 \mathrm{~dB}$ SFDR is attained. The total power consumption of the SC DAC is $90 \mathrm{~mW}$ with $1.2 \mathrm{~V}$ supply and clock frequency of $3 \mathrm{GHz}$.

In the fifth paper "Interpolating by a Factor of 3 in LowVoltage Low-Power Sigma-Delta DAC" Peter Pracný, Ivan Jørgensen and Erik Bruun presents the power optimization of a sigma-delta modulator based digital-to-ana$\log$ converter (DAC) for hearing aid audio back-end application. This new design freedom is used to lower the operating frequency of the whole back-end and save considerable amount of power. It is shown that the figure-ofmerit (FOM) of such designs can be lower than designs using oversampling by a factor of integer powers of two. The same optimization approach can be used for other low voltage low power portable audio applications.

In the sixth paper "A Low-Power 2nd-order CT Delta Sigma Modulator with a Single Operational Amplifier" authors Dejan Radjen, Martin Anderson, Lars Sundström and Pietro Andreani present a 2nd-order 4-bit continuous-time (CT) delta-sigma modulator (DSM) employing a 2nd-order loop filter with a single-operational-amplifier. This choice strongly reduces the power consumption, since operational amplifiers are the most power hungry blocks in the DSM. The DSM has been implemented in a $65 \mathrm{~nm}$ CMOS process, where it occupies an area of $0.08 \mathrm{~mm}^{2}$. It achieves a peak SNDR of $65 \mathrm{~dB}$ over a $500 \mathrm{kHz}$ signal bandwidth, while consuming $76 \mu \mathrm{W}$ from a $800 \mathrm{mV}$ power supply.

In the seventh paper "Design and Implementation of All MOS Micro Power Voltage Reference" authors Shailesh Singh Chouhan and Kari Halonen are proposing an all MOS voltage reference, which utilizes the classical principle of addition of two voltages with opposite temperature coefficients. The targeted application of the proposed voltage reference is a low dropout regulator which is used in a RF energy harvesting system. The proposed voltage reference has been implemented using standard $0.18 \mu \mathrm{m}$ CMOS technology. It generates reference voltage of $543.65 \mathrm{mV}$ with temperature coefficient of $17.43 \mathrm{ppm} /{ }^{\circ} \mathrm{C}$ for the temperature range of -40 to $+85{ }^{\circ} \mathrm{C}$.

In the eighth paper "An Integrated High Voltage Digitalto-Analog Converter for a Reconfigurable Antenna Array" authors Jing Ning, Lufei Shen, Erick González Rodríguez and Klaus Hofmann presents an integrated high voltage digital-toanalog converter, which is fabricated in a $0.35 \mu \mathrm{m}$ high voltage CMOS technology (AMS H35), and can be applied in high voltage applications up to $115 \mathrm{~V}$. The high voltage output is controlled by 8-bit digital input code. In order to improve the accuracy and decrease the required area, it is implemented by a low voltage DAC and a high voltage amplifier for boosting the controllable output voltage. Since the current consumption from the high voltage power supply is only $80 \mu \mathrm{A}$, it can be powered by a charge pump which generates $115 \mathrm{~V}$ from a battery with $3.7 \mathrm{~V}$ DC. Combining with a $8 \times 8$ external high voltage switch array, the proposed high voltage digital-to-analog converter can drive up to 64 individual channels of antenna array with a voltage from 0 to $115 \mathrm{~V}$. It will greatly reduce the complexity and cost of mobile applications which use tunable microwave devices and require high voltages. The feasibility is proved by post-simulation result and experimental test result. The proposed concept of the high voltage ASIC is also proved by a demonstrator. 
The last paper selected from the NORCHIP 2013 conference "Analysis of Band-to-band Mixing Distortion Contributions in Some Usual Circuit Topologies". In this paper authors Janne P. Aikio, Timo Rahkonen and Tero Korkala uses hand analysis and a new fully numerical distortion contribution analysis technique to explain and optimize the nonlinear performance of analog circuits. Several example circuits are studied, where mixing of nonlinear distortion from one harmonic band to another is important. In some circuits the band-to-band mixing can be employed to reduce the overall distortion.

The nine papers presented in this special issue have all been subject to the standard review process of the Journal.

Acknowledgments The guest editor want to express greatest appreciation to all the authors who submitted papers to the conference. As the time allowed for the selection, preparing and reviewing of the papers has been short I would like to thank the reviewers for doing through reviews and providing many useful comments and constructive criticism helping improving the quality of the papers. Also, I would like to thank the authors of selected papers for the responsiveness to the reviewers' comments and suggestions.

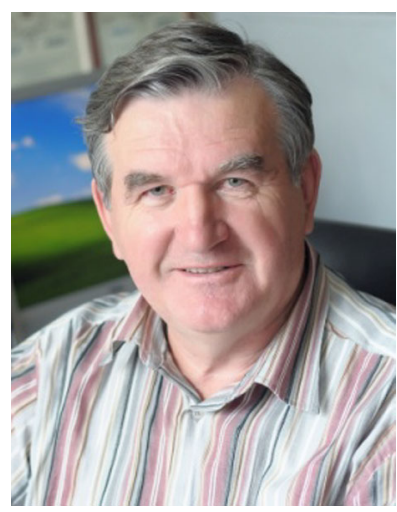

Romualdas Navickas received the electronics engineer degree at Kaunas Polytechnical Institute, in 1973; candidate of technical science (CTS) degree of USSR from Electronics Institute, Minsk, Belorussia, in 1984; Doctor of technical sciences degree from Lithuanian Council of Science (nostrified CTS), in 1993; Doctor Habilitus of technological sciences, in 2003; professor Vilnius Gediminas technical university, in 2004. Engineer, lead engineer, head laboratories of GaAs and Si technologies in Research Institute of
Microelectronics "Venta" in 1975-1999. Head or deputy in more 20 research projects of creation $\mathrm{Si}$ and $\mathrm{GaAs}$ integrated circuits and technologies, in 1976-1990, member of chief constructors board GaAs ICs of the USSR Electronics industry ministry, in 1987-1990. Scientific work supervisor of GaAs charge sensitive amplifiers design and manufacture for investigation high energy elementary particles physics in International Centre for Scientific Culture World Laboratory Lithuanian department in projects for CERN, Geneva, in 1992-1997. Expert and member of the board of the Lithuanian High Technology Development Program "Nanotechnologies and electronics", in 2005-2012. Representative Lithuanian Member in SSC of the European technological platform of nanoelectronics ENIAC, in 2005-2010. Member of the board Lithuanian association of "National Electronics and Nanoelectronics Cluster" in 2006 up to now. Leading person of micro-and nanoelectronics systems design and testing in the Lamatech national program in Sunrise Valley, Vilnius, responsible for creation laboratory of design of micro and nanoelectronic systems in project "Creation national centre of physical and technological sciences" (the budget about 63,5 mln. Euros), in 2009-2014. His current research interests are in the field of micro-and nano-electronics, highspeed integrated circuits design, nano-and microstructures self-formation processes. He is member of the IEEE.

Dr Habil Romualdas Navickas is author 4 books, he has made more than 80 publications, has 8 patents. 